\section{Fusion imaging of real-time ultrasonography with CT or MRI for hepatic intervention}

\section{Min Woo Lee}

Department of Radiology and Center for Imaging Science, Samsung Medical Center, Sungkyunkwan University School of Medicine, Seoul, Korea

With the technical development of ultrasonography (US), electromagnetic tracking-based fusion imaging of real-time US and computed tomography/magnetic resonance (CT/MR) images has been used for percutaneous hepatic intervention such as biopsy and radiofrequency ablation (RFA). Because of the fusion imaging technique, the fused CT or MR images show the same plane and move synchronously while performing real-time US. With this information, fusion imaging can enhance lesion detectability and reduce the false positive detection of focal hepatic lesions with poor sonographic conspicuity. Three-dimensional US can also be fused with realtime US for the percutaneous RFA of liver tumors requiring overlapping ablation. When fusion imaging is not sufficient for identifying small focal hepatic lesions, contrast-enhanced US can be added to fusion imaging.

Keywords: Fusion imaging; Hepatocellular carcinoma; Radiofrequency ablation; Biopsy; Liver

\section{Introduction}

Ultrasonography (US) has been widely used for interventional procedures for the liver due to a number of advantages: real-time capability, no radiation hazard, easy accessibility, and low cost $[1,2]$. When performing US-guided interventional procedures, the operators need to mentally register the reference data set (computed tomography [CT] or magnetic resonance [MR] images) and the working data set (real-time US images) [3]. However, this mental registration can be challenging when the liver cannot be scanned with orthogonal transverse, sagittal, or coronal planes, which are frequently used for the interpretation of CT or MR images in our daily practice. In addition, the deformation and displacement of the liver occurs by the breathing motion and heartbeats of patients. Moreover, a sonographic window of the liver is sometimes limited by the rib cage, colon, or omental fat surrounding the liver. Therefore, if erroneous mental registration occurs, this may lead to mistargeting during US-guided hepatic interventions [4].

Fusion imaging is a technique that fuses two different imaging modalities. In the field of hepatic intervention, real-time US is usually fused with other imaging modalities such as CT, MR, and positron emission tomography (PET)/CT. In our daily practice, challenging small target lesions are more frequently detected than before due to recent advances in CT and MR technology. Therefore,

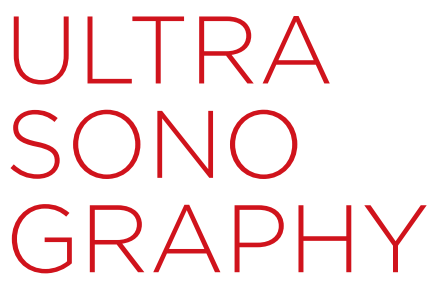

REVIEW ARTICLE

http://dx.doi.org/10.14366/usg. 14021 pISSN: 2288-5919 - elSSN: 2288-5943

Ultrasonography 2014;33:227-239

Received: April 21, 2014

Revised: June 9, 2014

Accepted: June 10, 2014

Correspondence to:

Min Woo Lee, MD, Department of Radiology and Center for Imaging Science, Samsung Medical Center, Sungkyunkwan University School of Medicine, 81 Irwon-ro, Gangnam-gu, Seoul 135-710, Korea

Tel. +82-2-3410-2548

Fax. +82-2-3410-0049

E-mail: leeminwoo0@gmail.com

This is an Open Access article distributed under the terms of the Creative Commons Attribution NonCommercial License (http://creativecommons.org licenses/by-nc/3.0/) which permits unrestricted noncommercial use, distribution, and reproduction in any medium, provided the original work is properly cited.

Copyright (C) 2014 Korean Society of Ultrasound in Medicine (KSUM)

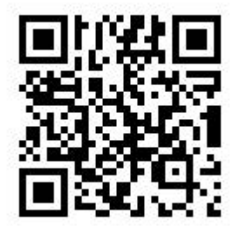

How to cite this article:

Lee MW. Fusion imaging of real-time ultrasonography with CT or MRI for hepatic intervention. Ultrasonography. 2014 Oct; 33(4):227-239. 
fusion imaging has gained considerable attention since it can help operators conduct interventional procedures with high confidence and accuracy for challenging target lesions $[5,6]$. In this article, an electromagnetic (EM) tracking-based fusion imaging technique and its current clinical application for the hepatic intervention is reviewed.

\section{Electromagnetic Tracking-Based Image Fusion}

For the fusion imaging technique, a variety of tracking methods are available, including optical, image-based, and EM tracking [3]. In general, optical tracking is most commonly utilized for surgical procedures and image-based tracking for vascular interventions. In contrast, EM tracking is most widely used for US-guided hepatic interventions. There are three components of the EM tracking-based fusion imaging technique: the magnetic field generator, position sensor, and position sensor unit. The magnetic field generator, which is located near the patient, creates a magnetic field, thereby inducing currents in the position sensor, which is mounted on the US transducer. As the US probe moves, the magnitude of the electrical current in the position sensor changes with respect to the magnetic field. With this information, the position sensor unit installed in the US machine calculates the exact location of the position sensor and thus, determines the direction and position of the US transducer $[3,7]$. This enables the side-by-side or overlay display of real-time US images and fused CT or MR images.

Image fusion by EM tracking can be performed by using either external fiducial markers or internal anatomic landmarks. When CT is performed with external fiducial markers attached to the body surface around a target organ, image fusion can be performed automatically between real-time US images and CT images. This is because the fiducial markers contain position sensor coils and are radio-opaque on CT images [8]. External MRI fiducial markers would also be helpful for accurate image fusion between real-time US and MR images. However, they are under development and are rarely available for EM tracking-based fusion imaging.

Image fusion can also be performed using the internal markers of patients. Internal markers refer to anatomic landmarks within a patient's liver, such as focal hepatic lesions (i.e., cyst or calcification), bifurcation of the hepatic or portal vein, or liver configuration [9]. Despite several differences among various vendors, the basic concept of image fusion using internal markers is similar. The first step of image fusion using this method is uploading the data set to be fused with real-time US to the US machine. Then, image fusion is performed, which generally consists of plane and point registration. Plane registration is a process of finding the same plane on realtime US and uploaded CT or MR images. Any plane that clearly shows anatomic landmarks on both data sets can be used for plane registration. After plane registration, point registration is performed to match the two image data sets more accurately. This is usually performed by pointing out the same anatomic point near the target lesion between real-time US and CT or MR images, which can be repeated until optimal image fusion is obtained (Fig. 1). If a target lesion is already identified on real-time US before applying fusion imaging, image fusion can be performed very quickly using the following method: the image plane containing the target lesion is used for plane registration, and the target lesion itself is used for point registration by indicating the center of the lesion.

Instead of repeated point registrations, other vendors have a "rotate" or "drag" function to match the real-time US and uploaded CT or MR images, which is usually performed on the overlay display of the two data sets. There is also manual image fusion, which is performed by matching three internal markers on any image plane, including the oblique plane.

After the application of the fusion imaging technique, real-time US and fused CT or MR images appear side-by-side or overlaid on the US monitor. Therefore, the fused CT or MR image shows the same plane and moves synchronously with real-time US (Video clip 1). With the information of the fused CT or MR images, the location of the target lesion in relation to the perilesional anatomic landmark is easier to recognize on real-time US. Therefore, fusion imaging allows operators to detect a target lesion with poor sonographic conspicuity.

The time required for image fusion varies depending on the fusion imaging technologies and the level of experience of the operators. Since image fusion using internal markers may be cumbersome for beginners, automatic image fusion between real-time US and preprocedural CT or MR images would be helpful to simplify the overall workflow. Although prototype automatic image fusion by sweeping the liver volume has been introduced [10], clinical data for hepatic intervention with this automatic registration are rarely available. However, automatic registration is expected to be helpful for less experienced operators with respect to manual registration $[11,12]$.

According to ex vivo experimental studies [13,14], the registration error between real-time US and CT images was within $3 \mathrm{~mm}$, and the targeting error was also about $3 \mathrm{~mm}$. Although these studies were performed in the explanted liver, the error seems to be acceptable.

\section{Liver Application of Fusion Imaging}

Many hepatic interventional procedures benefit from fusion imaging, enabling operators to conduct percutaneous interventional procedures for challenging target lesions that would otherwise 


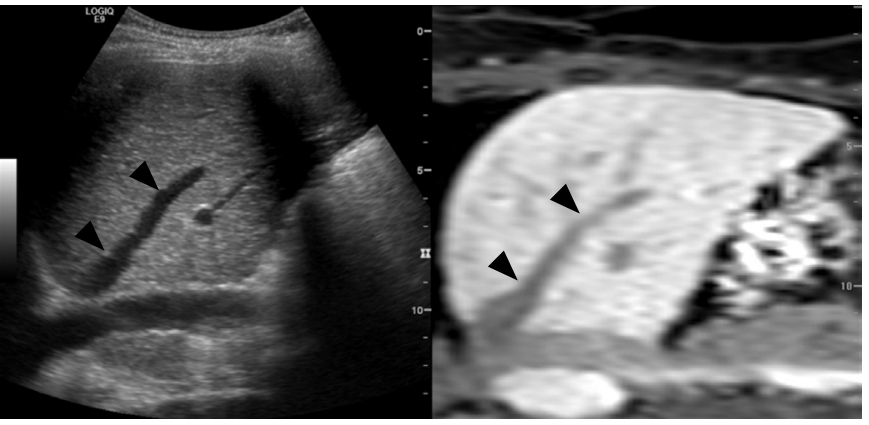

A

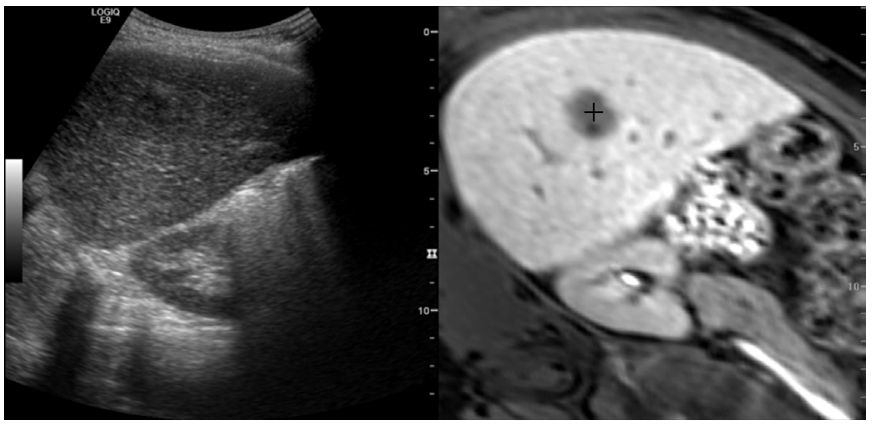

C

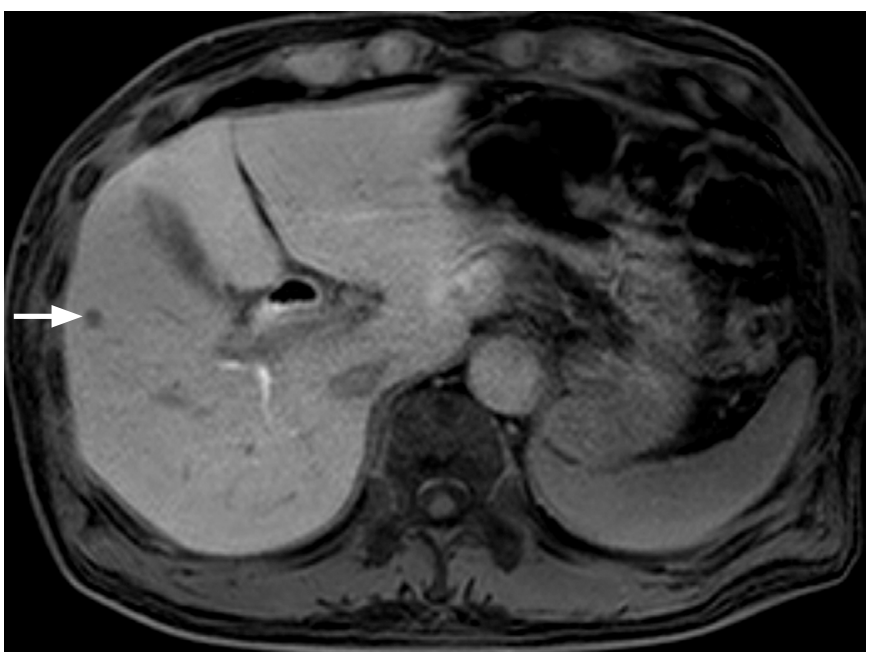

A

Fig. 2. Fusion imaging-guided biopsy of a focal hepatic lesion with poor sonographic conspicuity in a patient with pancreatic head cancer.

A. Hepatobiliary-phase magnetic resonance (MR) image shows a 6-mm focal hepatic lesion (arrow) in the liver. B. Percutaneous biopsy was performed to rule out metastasis. The lesion (arrow) can be identified with the use of fusion imaging even though it was invisible on B-mode ultrasonography. Biopsy revealed that it was a metastasis from pancreatic cancer. 
not be possible $[5,6,9,15-22]$. For example, fusion imaging is useful for the percutaneous biopsy of a focal hepatic lesion that has poor conspicuity on B-mode US $[6,23,24]$ (Figs. 2, 3). Using this technique, we can reduce the sampling error by an accurate localization of the target lesions.

Fusion imaging also improves the conspicuity of hepatocellular carcinomas (HCCS) and the feasibility of percutaneous radiofrequency ablation (RFA) of HCCs not identifiable on B-mode US $[9,18]$. Therefore, it can reduce the number of RFA sessions for HCCS invisible on B-mode US [18]. Given that the detectability of HCCS depends on the tumor size $[1,25]$, fusion imaging is likely to be more useful for small HCCs. This assumption was supported by a recent study where the detectability of HCCs smaller than $2 \mathrm{~cm}$ was much higher when using fusion imaging than when using conventional B-mode US, whereas for HCCs equal to or larger than $2 \mathrm{~cm}$, this was not the case [5]. Fusion imaging is also useful in avoiding the mistargeting of HCC-ablating a pseudolesion, not the true lesion-by reducing the false-positive detection of small HCCS on B-mode US $[5,22]$ (Fig. 4). Fusion imaging can also guide accurate overlapping ablations of HCCs on the basis of the fused CT or MR images (Fig. 5).

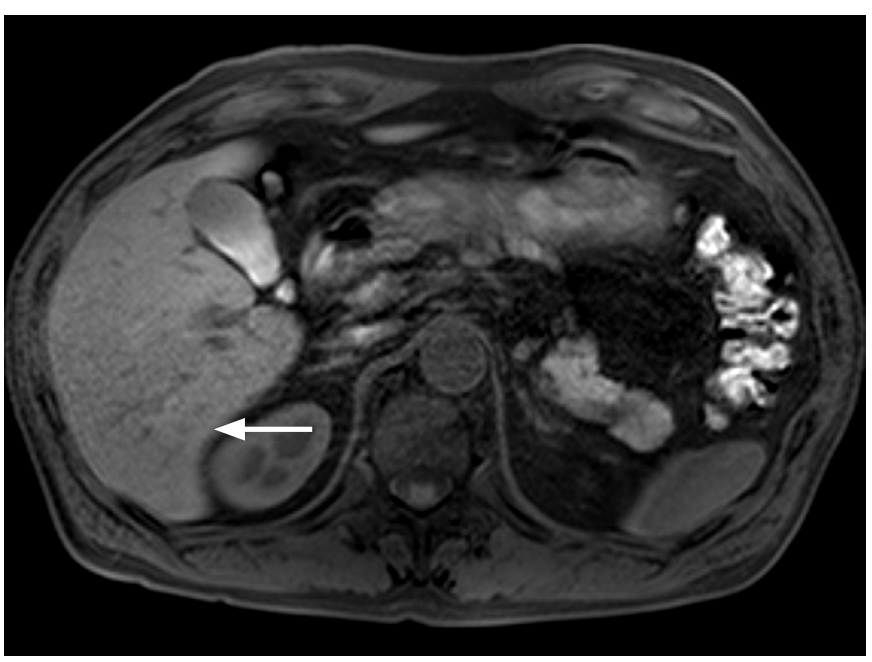

A

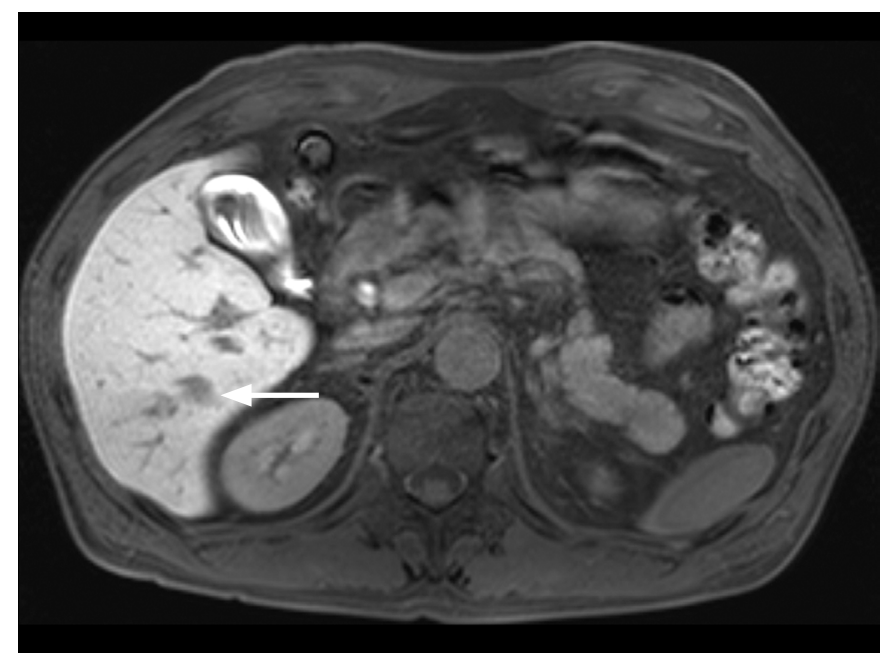

C

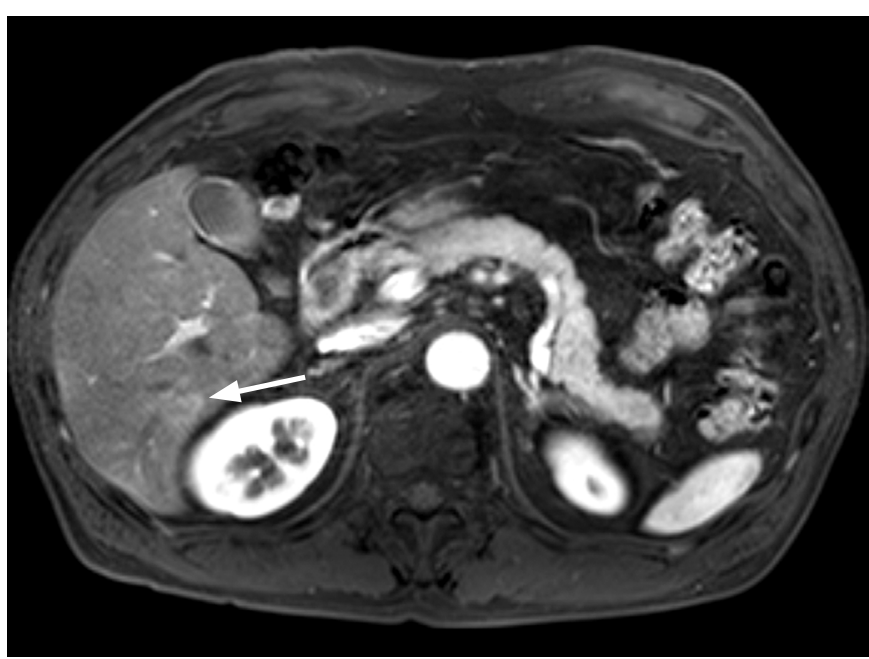

B

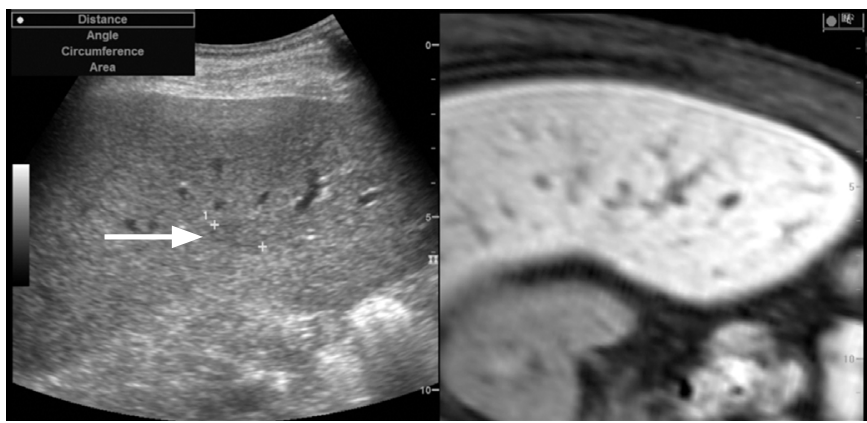

D

Fig. 3. Fusion imaging-guided biopsy of a focal hepatic lesion in a patient with hepatitis B virus carrier.

A. Precontrast T1-weighted magnetic resonance (MR) image shows an ill-defined lesion (arrow) with an iso-to-high signal intensity. B. The lesion (arrow) shows arterial enhancement. C. In the hepatobiliary phase, the lesion (arrow) shows a low signal intensity. D. Planning ultrasonography (US) for radiofrequency ablation (RFA) was performed since the lesion was diagnosed as a small hepatocellular carcinoma (HCC) on the MR images. On planning US with fusion imaging, the location (arrow) of the lesion on US was different from that on the fused MR image. In addition, since the signal intensity on the pre-contrast T1-weighted image was not consistent with that of the usual HCC, instead of RFA, percutaneous biopsy was performed; it revealed that the lesion was an eosinophilic abscess. 
However, not all small HCCs are visible on fusion imaging. The detection of small HCCs in a deep location in patients with liver cirrhosis is still challenging even with the guidance of fusion imaging. According to a recent study [9], out of $120 \mathrm{HCCs}$ (mean tumor size, $1.0 \mathrm{~cm}$; range, 0.5 to $2.2 \mathrm{~cm}), 82 \mathrm{HCCs}(68.3 \%)$ were invisible even on fusion imaging. However, $31.7 \%$ (26/82) of the HCCs unidentifiable on fusion imaging could be ablated by inserting an electrode on the basis of peritumoral anatomic landmarks.

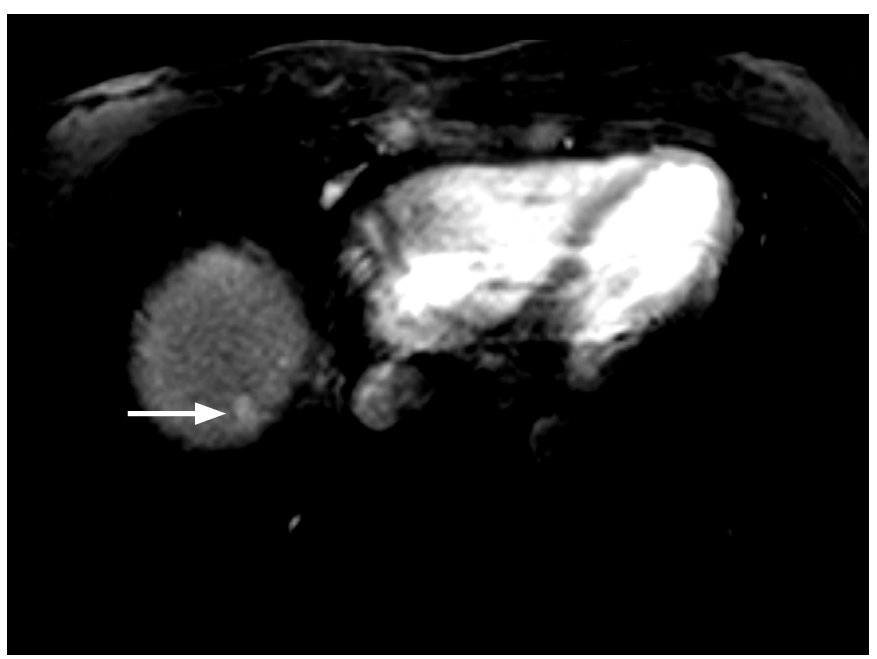

A

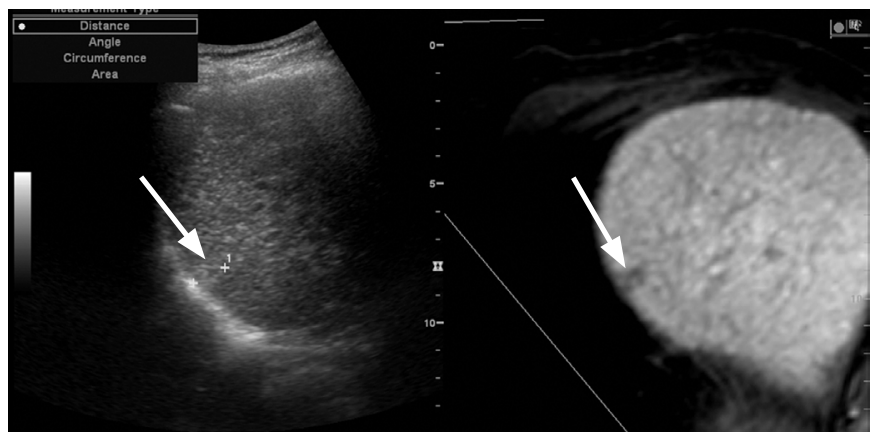

C

\section{Fusion Imaging with Contrast-Enhanced US}

Since contrast-enhanced ultrasonography (CEUS) is also useful for the guidance of percutaneous hepatic interventions [26-28], CEUS can be used in addition to fusion imaging if the target lesions have poor conspicuity even on fusion imaging (Figs. 6-8). When used simultaneously, CEUS is displayed with fusion imaging, not with B-mode US [29]. Among various US contrast agents, Sonazoid (perflubutane microbubbles; GE Healthcare, Oslo, Norway), offering both vascular and post-vascular phases, is effective in localizing

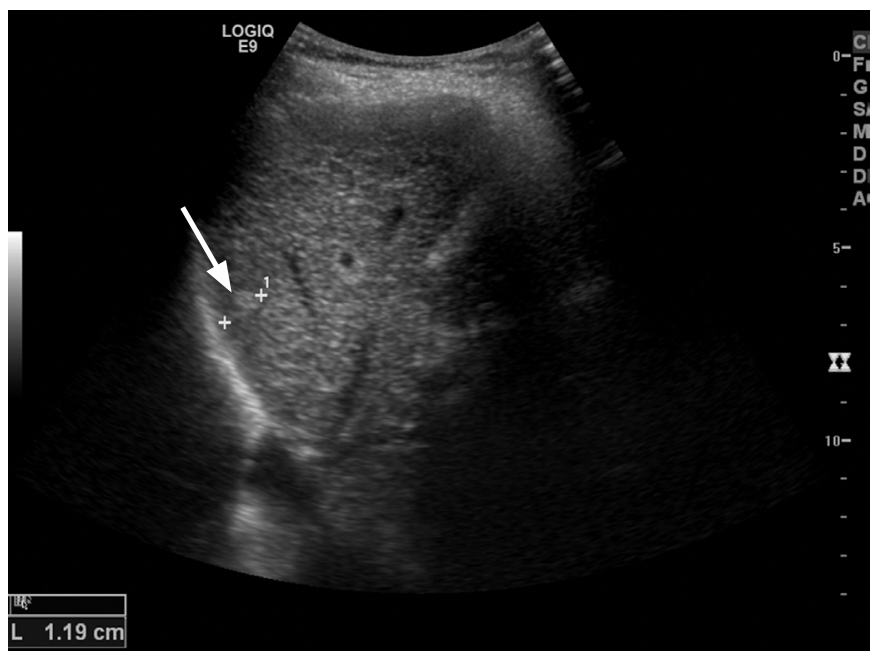

B

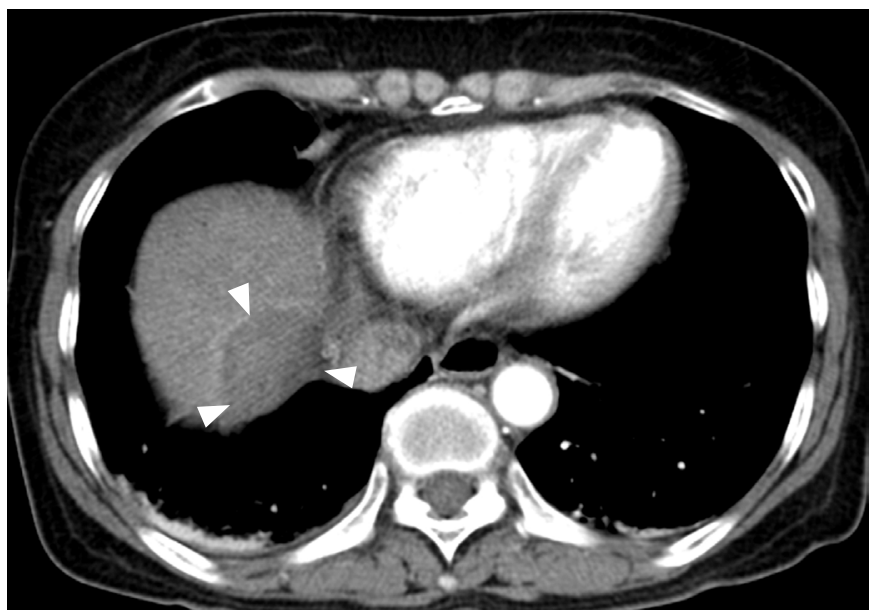

D

Fig. 4. Fusion imaging-guided radiofrequency ablation (RFA) of a 1.2-cm hepatocellular carcinoma (HCC) with poor sonographic conspicuity in a patient with a hepatitis B virus carrier.

A. Arterial-phase T1-weighted magnetic resonance (MR) image shows a small enhancing tumor (arrow) in the right liver dome. B. On planning ultrasonography (US) before image fusion, a small echogenic lesion (arrow) was identified and was considered to be the HCC identified upon MRI. C. However, after image fusion, the index tumor (arrow) was located at a different site. Therefore, the lesion identified before image fusion was a pseudolesion. Percutaneous RFA was performed with confidence. D. Immediate post-RFA computed tomography scan shows that it was a technical success with a sufficient ablative margin (arrowheads). In this case, mistargeting was avoided by using the fusion imaging technique. 
small HCCS that have poor conspicuity on B-mode US $[30,31]$.

The combined use of fusion imaging and CEUS has several advantages for localizing small HCCs with poor sonographic conspicuity. Most of all, the overall procedure time of CEUS-guided intervention can be reduced. With fusion imaging, the operator

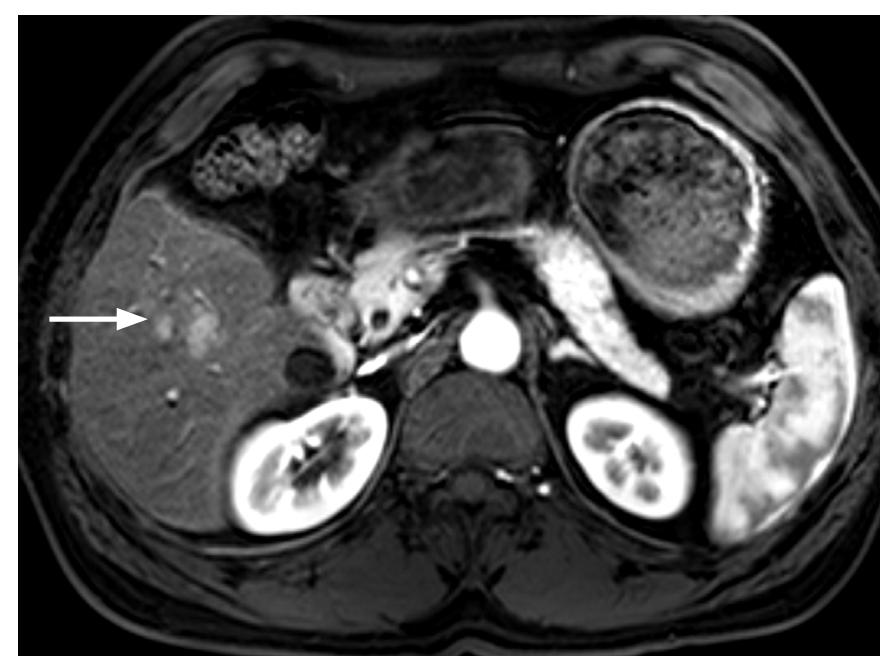

A

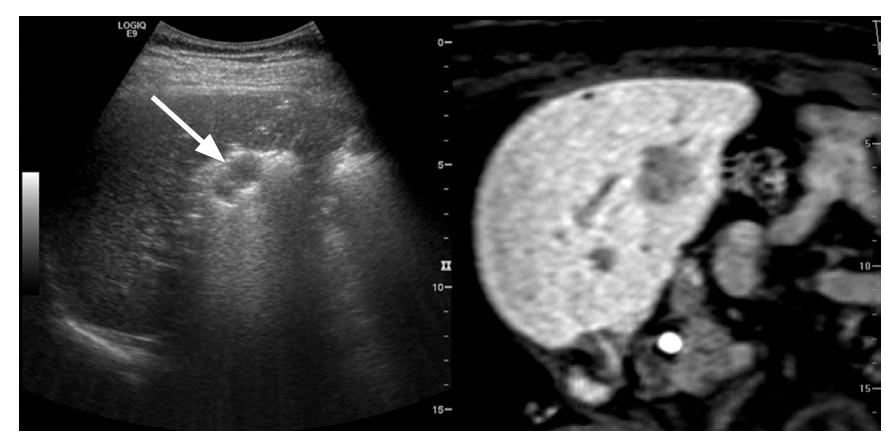

C

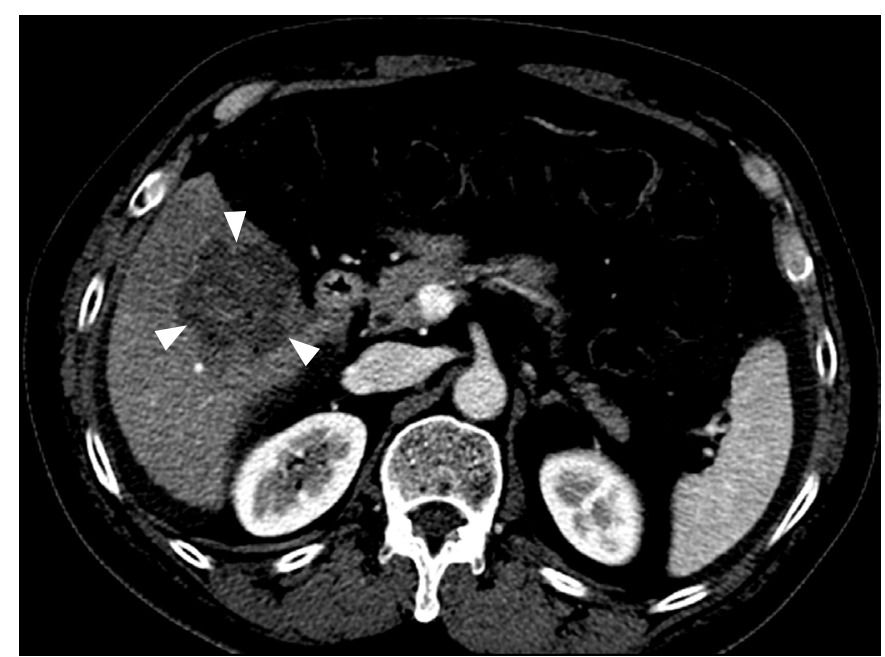

can estimate the location of the target lesion before contrast injection, making it easier to evaluate a nodule throughout the vascular phase. If the lesion shows arterial enhancement and a low echogenic defect during the postvascular phase, we do not have to re-inject Sonazoid to see the so-called defect-reperfusion imaging

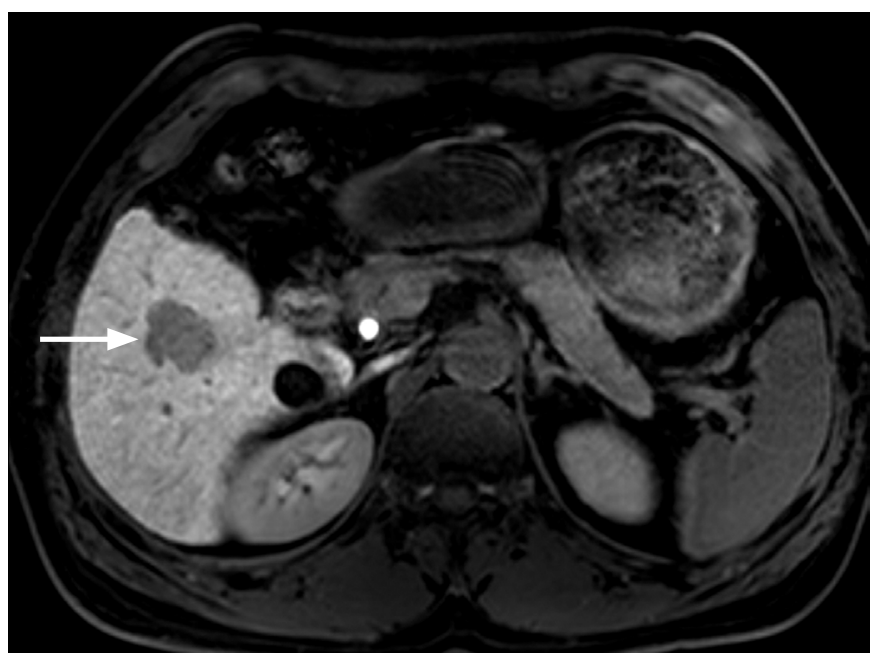

B

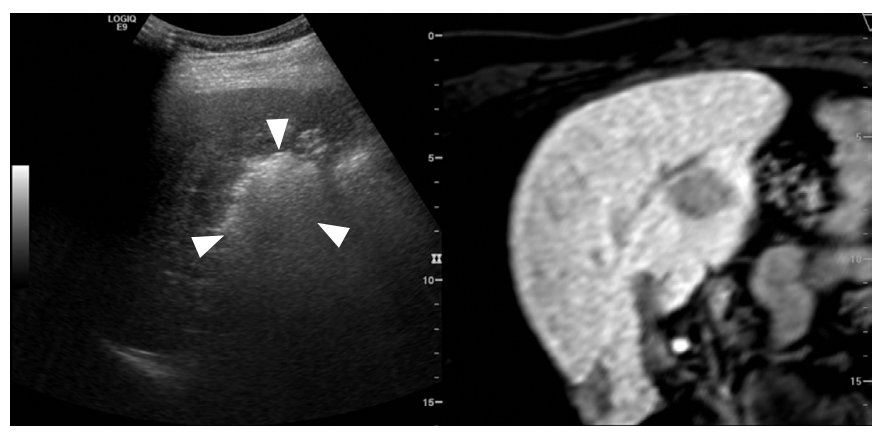

D

Fig. 5. Fusion imaging-guided radiofrequency ablation (RFA) of a 3.1-cm hepatocellular carcinoma.

A. Arterial-phase T1-weighted magnetic resonance (MR) image shows an enhancing tumor (arrow) in the right lobe of the liver. B. The lesion (arrow) shows a low signal intensity in the hepatobiliary phase. C. Fusion imaging-guided RFA was performed. Residual unablated tumor (arrow) was identified intuitively on the basis of the fused MR images. D. The residual tumor was ablated by repositioning one electrode. A large echogenic zone (arrowheads) was made, which was sufficiently large to cover the entire tumor. E. Immediate post-RFA computed tomography scan shows that it was a technical success with a sufficient ablative margin (arrowheads). 


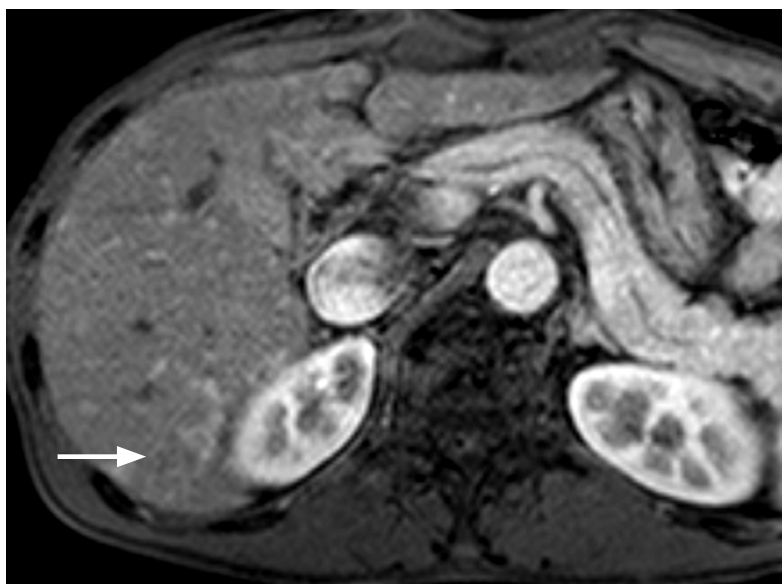

A

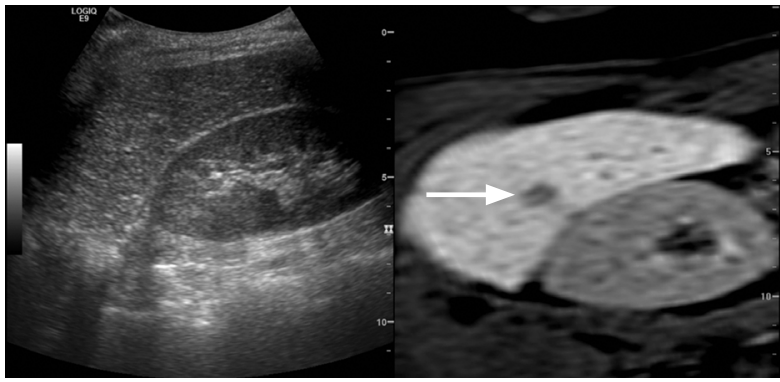

C

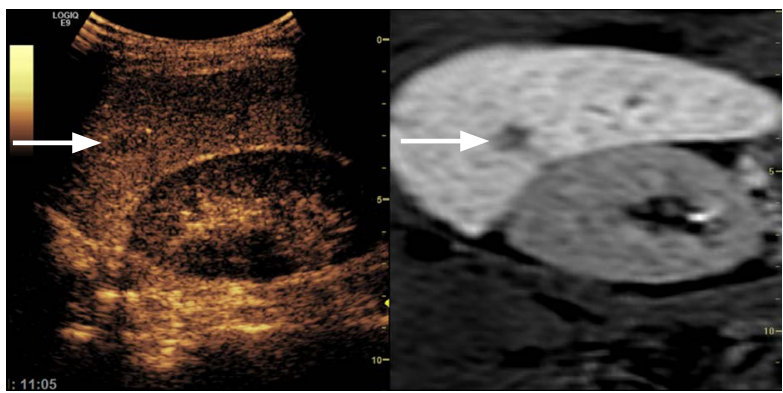

$\mathrm{E}$

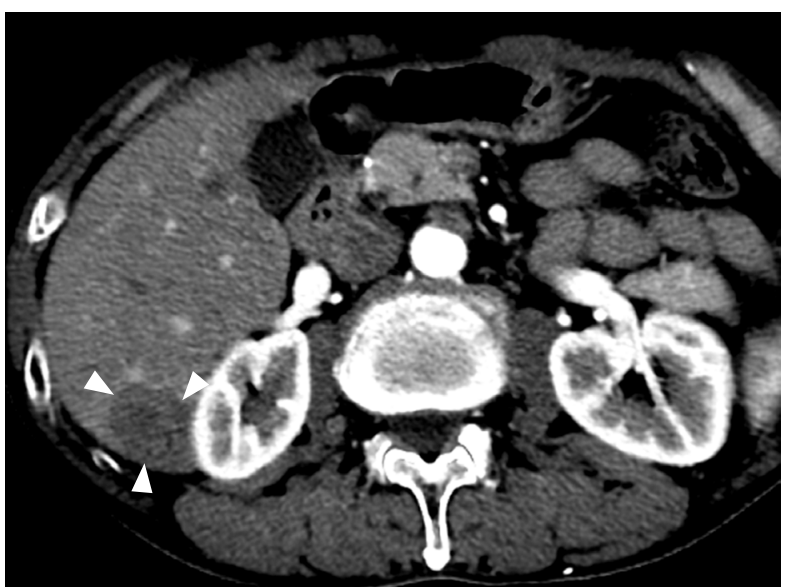

G

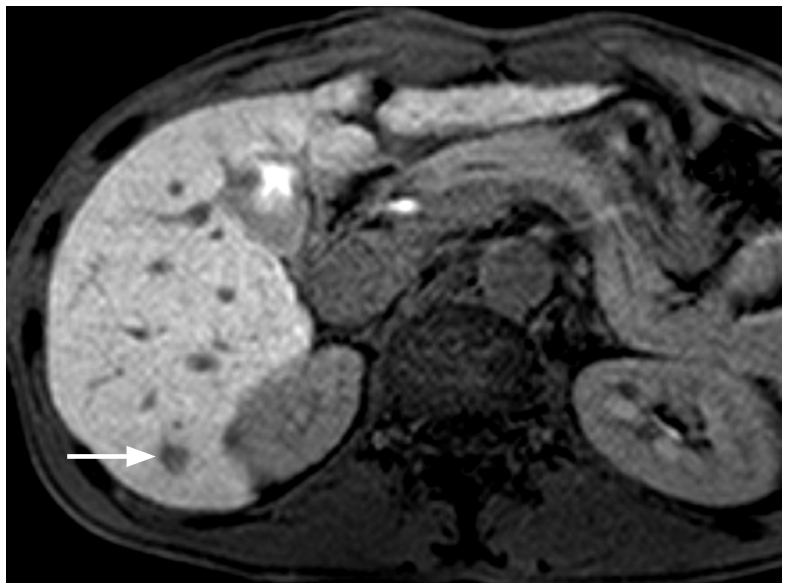

B

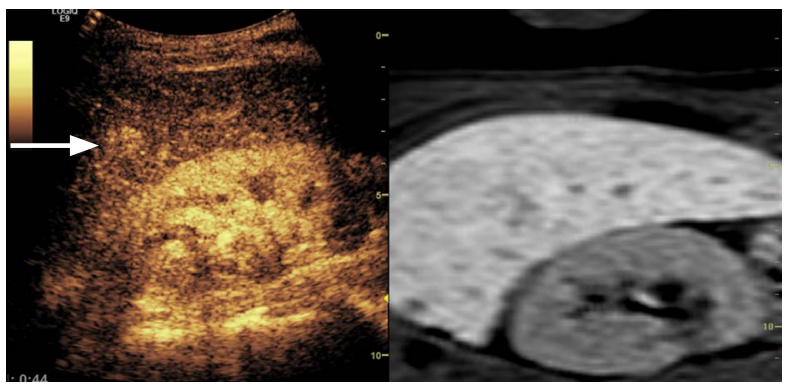

D

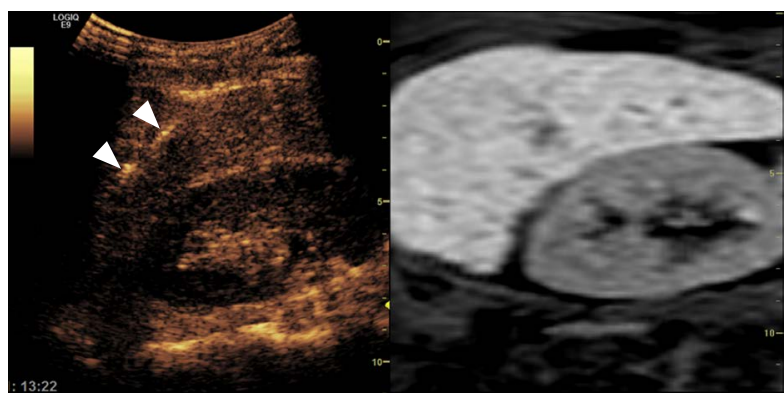

$\mathrm{F}$

Fig. 6. Fusion imaging and contrast-enhanced ultrasonography (US)guided radiofrequency ablation (RFA) of a $1-\mathrm{cm}$ hepatocellular carcinoma (HCC) in a patient with a hepatitis B virus carrier.

A. Arterial-phase T1-weighted magnetic resonance (MR) image shows a subtle enhancing lesion (arrow) in segment 6 of the liver. B. The lesion (arrow) shows a low signal intensity in the hepatobiliary phase. C. Fusion imaging-guided RFA was performed. Due to the coarse echo texture of the liver, the index tumor was unidentifiable even on the US of fusion imaging where the tumor (arrow) was located in the fused MR image. D. Contrastenhanced US was added to the fusion imaging. In the arterial phase, an enhancing nodule (arrow) is identified. E. In the Kupffer phase, the lesion (arrow) is seen as a defect, confirming that the lesion is an HCC. F. Targeting was performed in the Kupffer phase. Arrowheads indicate the exposed tip of an electrode. G. Immediate post-RFA computed tomography scan shows that the tumor was completely covered by the ablation zone (arrowheads), suggestive of technical success. 


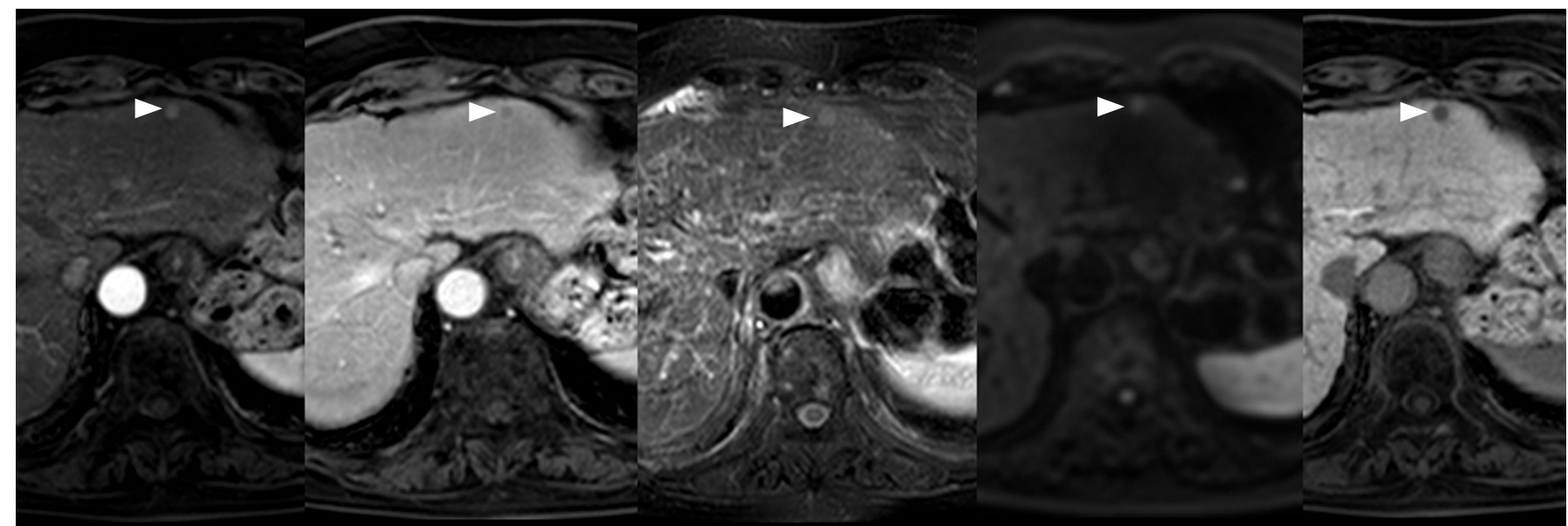

A

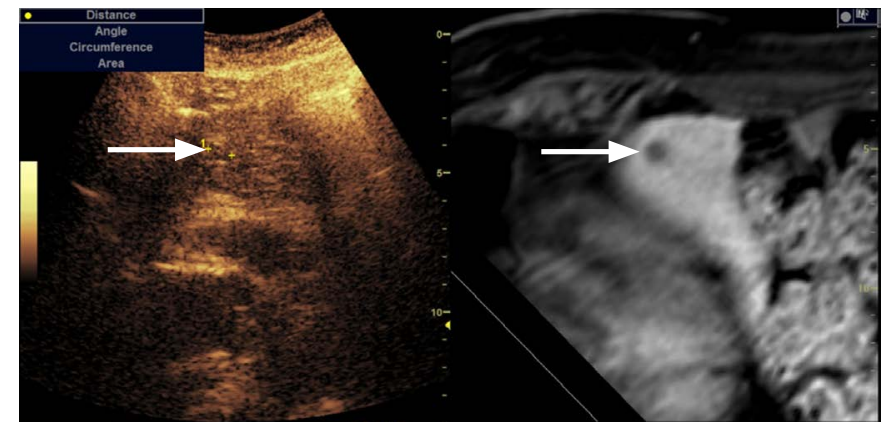

B

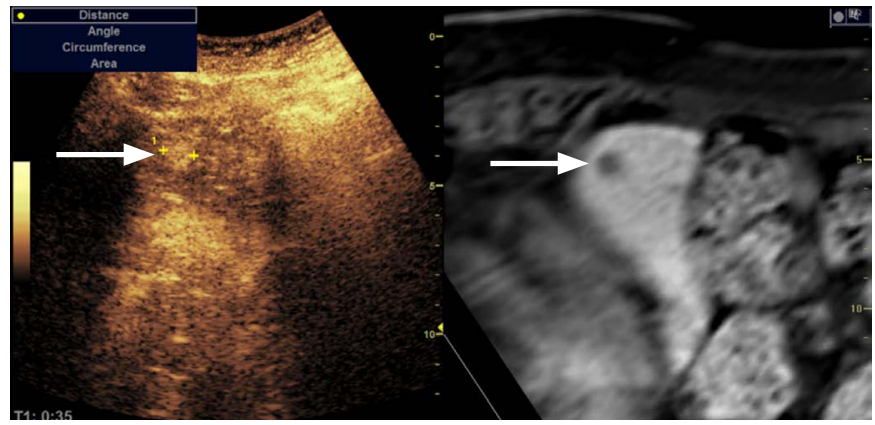

C

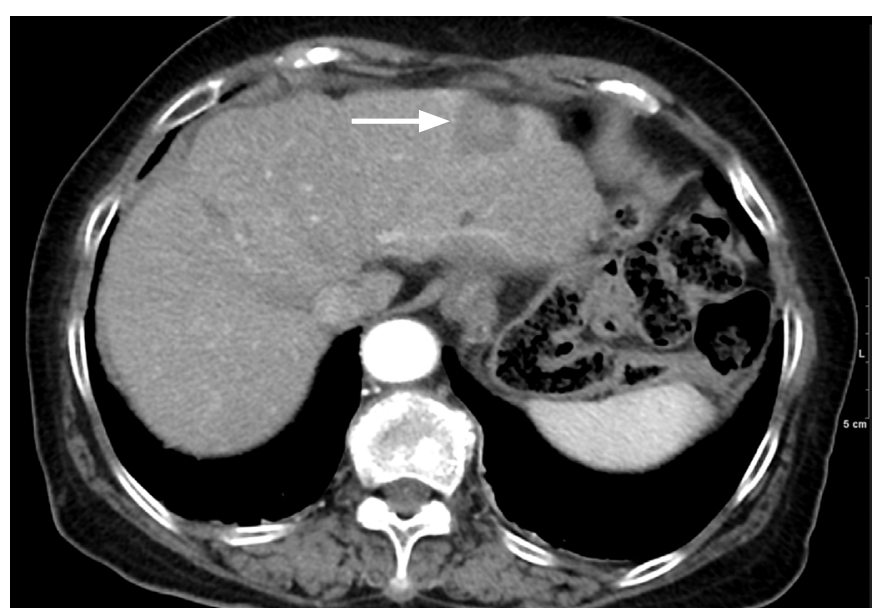

E

D

Fig. 7. Fusion imaging and contrast-enhanced ultrasonography (US)-guided radiofrequency ablation (RFA) of a subcentimetersized recurrent hepatocellular carcinoma (HCC) in a patient with a hepatitis B virus carrier. The patient had previously undergone percutaneous RFA of HCC.

A. In the magnetic resonance (MR) images (arterial phase, followed by portal phase, T2-weighted image, diffusion-weighted image, and hepatobiliary phase), the lesion (arrowheads) shows the typical imaging features of HCC. Although the size of the lesion was measured to $0.8 \mathrm{~cm}$, it was regarded as a recurrent HCC since it was a new lesion, not seen in the previous computed tomography or MR images. B. To be certain, contrast-enhanced US was performed in addition to the fusion imaging. On the scout image, the lesion (arrow) was identified at the same location where the index tumor (arrow) was located on the fused MR image. C. In the arterial phase, the lesion (arrow) shows strong enhancement. D. In the Kupffer phase, the lesion (arrow) is seen as a defect, indicating that the lesion is an HCC. E. Immediate post-RFA computed tomography scan shows that the tumor was completely covered by the ablation zone (arrow), suggestive of technical success. 


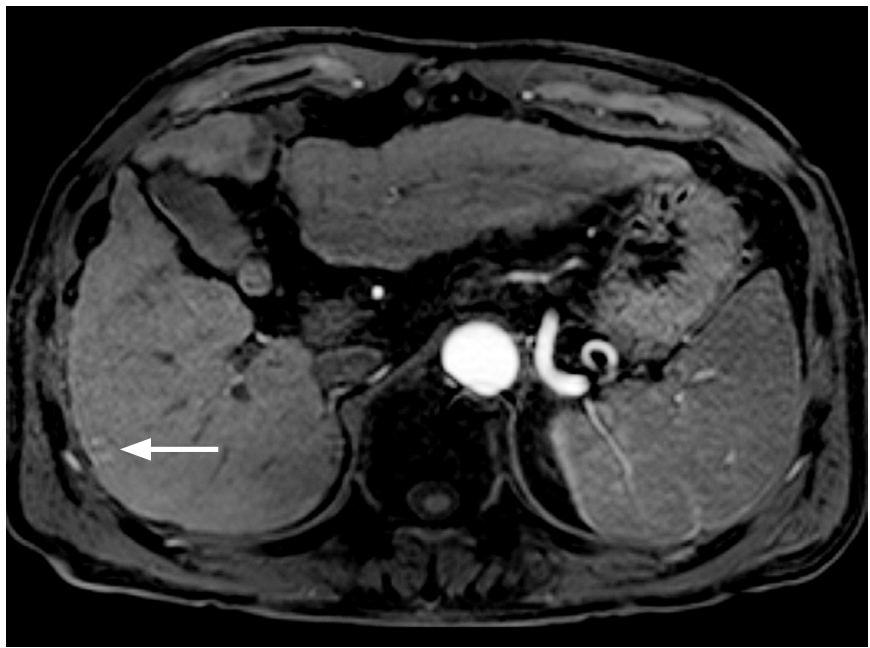

A

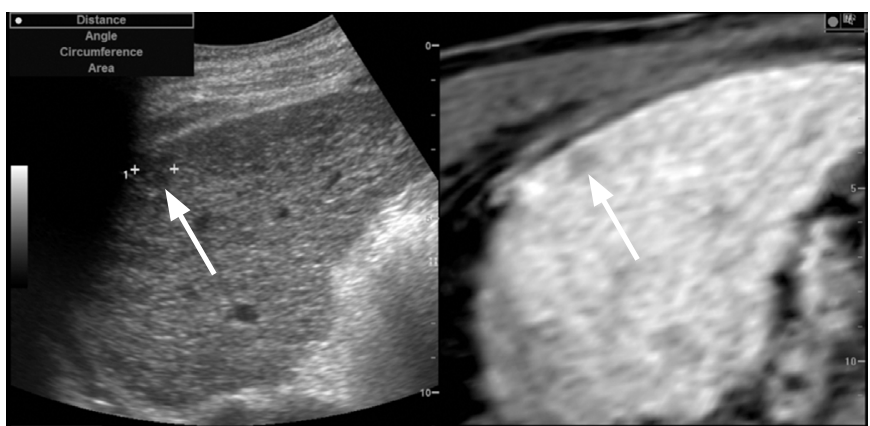

C

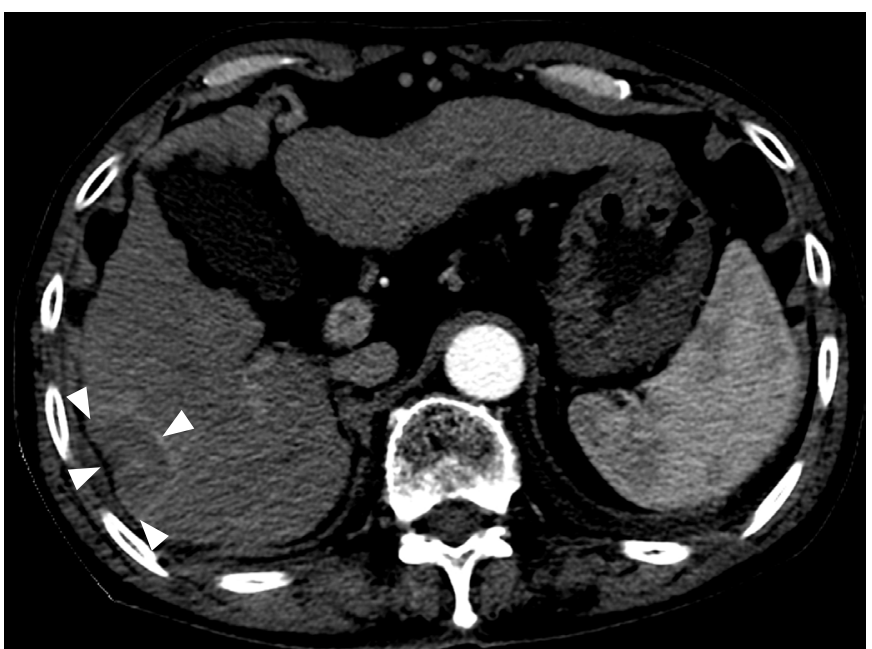

E

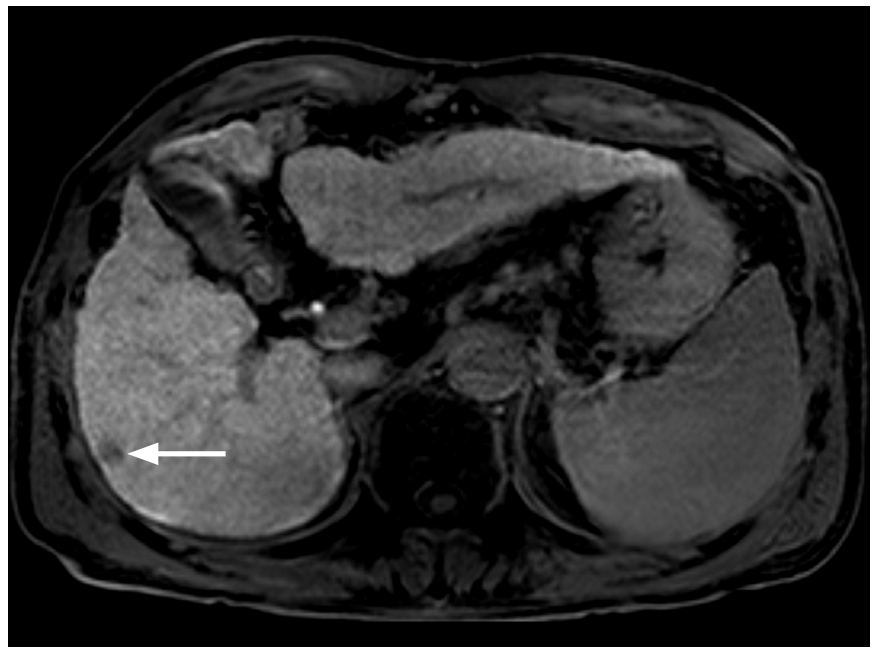

B

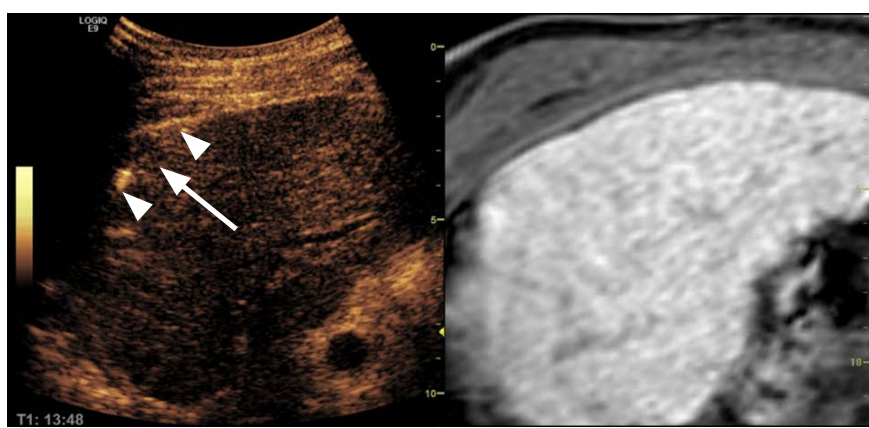

D

Fig. 8. Fusion imaging and contrast-enhanced ultrasonography (CEUS)-guided radiofrequency ablation (RFA) of a $1.3-\mathrm{cm}$ hepatocellular carcinoma (HCC) in a patient with a hepatitis $B$ virus carrier.

A. Arterial-phase T1-weighted magnetic resonance (MR) image shows a small enhancing lesion (arrow) in the periphery of segment 6 of the liver. B. The lesion (arrow) shows a low signal intensity in the hepatobiliary phase. C. Fusion imaging-guided RFA was performed. On the US of the fusion imaging, a small low echoic lesion (arrow) was identified at the similar location (arrow) of the tumor on the fused MR image. D. However, to obviate the risk of mistargeting, CEUS was performed additionally. On CEUS, the lesion identified on fusion imaging (C) did not show arterial enhancement. True HCC was obscured by a rib shadow and was identified as a small enhancing lesion when the patient breathed in slightly when the liver was slightly shifting down (not shown here, Video clip 3). The lesion was targeted with the patient holding the breath after inhaling a small amount of air in the Kupffer phase when the index tumor (arrow) was seen as a defect. The arrowheads indicate the exposed tip of the electrode. E. Immediate post-RFA computed tomography scan shows that the tumor was completely covered by the ablation zone (arrowheads), suggestive of technical success. In this example, mistargeting was avoided by the use of CEUS in addition to fusion imaging. 
for HCC [32]. Therefore, the overall procedure time of CEUS-guided intervention would be substantially decreased if CEUS is combined with fusion imaging [33].

\section{Image Fusion with 3-Dimensional US Images}

Three-dimensional (3D) US can also be used as a reference data set and can be fused with real-time US. Unlike CT or MR images, which are obtained prior to the RFA procedure, 3D US images are obtained at the time of RFA procedures after electrode insertion. If $3 D$ US is fused with real-time US, it can help the operator to monitor and control RFA procedures. On the basis of the fused 3D US, the operator can understand comprehensive 3D relationships of the index tumor, peritumoral anatomic landmarks, and electrodes inserted through the tumor. The information with respect to the location of the electrode in relation to the tumor makes electrode repositioning more convenient whenever it is needed (Video clip 2).

\section{Electromagnetic Needle Tracking}

Virtual needle tracking can be provided by the fusion imaging system with the use of the EM position sensor embedded in the distal tip of a needle or attached to the hilt of the needle $[34,35]$. Theoretically, the accuracy of virtual needle tracking depends on the location of the EM position sensor. A needle with a position sensor at the distal tip of the needle can provide a more accurate expected electrode path than that with a position sensor attached to the hilt of the needle because the needle can bend in the liver at the time of targeting $[34,36]$. This fact was supported by a study in which the virtual tracking error was less than $2 \mathrm{~mm}$ when the position sensor was located at the distal tip of the needle [34]. A virtual needle tracking system would be valuable for interventional procedures for challenging target lesions. For example, target lesions that cannot be reached by the usual in-plane approach would benefit from a needle tracking system that can allow operators to reach the target lesion with an out-of-plane approach [36,37]. In addition, large tumors requiring overlapping ablation would benefit from the needle tracking system since the virtual electrode tip appears within an echogenic cloud during the ablation procedures [35].

\section{Limitation of Fusion Imaging}

Since the usual reference data sets (CT, MRI, or PET/CT) are obtained with the patients in a breath-holding state, they contain static images. In comparison, a working data set (real-time US) is affected by tissue deformation due to the patient's respiration and movement. In addition, the positioning of patients during a real- time US examination can differ from that during the acquisition of reference data sets. Moreover, most commercially available fusion imaging systems are based on rigid registration, which lacks compensating patient respiration and movement [7]. Hence, some degree of registration error is inevitable between real-time US and fused reference data sets. This assumption is supported by the results of a previous study [38] in which the mean maximum registration error between real-time US and fused CT images was $11.5 \mathrm{~mm}$ in patients with hepatic metastasis. To decrease the registration error, it would be better to perform image fusion when the respiratory status of a patient is the same as when the reference data set was acquired. For example, if the MR images to be fused with real-time US was obtained during the expiratory phase, image fusion should be performed during the same expiratory state of the patient.

In a recent study [39], mistargeting occurred in 1.3\% (7/551) of the patients with HCCs even though fusion imaging was used for the guidance of RFA of HCCs. All patients who suffered from mistargeting were hepatitis $B$ virus (HBV) carriers, and their tumors were mostly smaller than $1.5 \mathrm{~cm}$ and were located in the periphery of the liver. Given that liver cirrhosis caused by HBV is characterized by macronodular cirrhosis, which has numerous pseudolesions mimicking the target tumor on the US examination [4], caution should be taken during RFA procedures in patients who are HBV carriers. In addition, when the tumor is located in the periphery of the liver, it may not be possible to use large vessels as anatomic landmarks to locate the tumor. Moreover, liver deformation and displacement by the patient's breathing motion may be more apparent in the peripheral than in the central liver [39]. Therefore, for these challenging cases with a small size and a peripheral location, CEUS can be combined with fusion imaging to obviate the risk of mistargeting (Fig. 8, Video clip 3).

In the EM tracking-based fusion imaging technique, increased magnetic field strength can increase the accuracy of image fusion. Hence, the magnetic field generator should be located near the region of interest and skin fiducials, which may restrict free device movement during the procedures. In addition, since magnetic field homogeneity is also crucial, any conductive object or magnetic substance should be located far away so as not to alter the magnetic field $[3,40]$.

\section{Future of Fusion Imaging}

Since the manual fusion of real-time US and CT or MR images is difficult for beginners, most vendors are developing automatic image fusion methods. However, they need more improvements. First of all, the speed of image fusion and its accuracy should be 
improved further. In addition, the success rate of automatic image fusion should be enhanced. Furthermore, although automatic image fusion between real-time US and preprocedural CT images has been introduced by some vendors, it should also work with preprocedural MR images. In general, MR images are preferred as the reference data set over $\mathrm{CT}$ images due to the high contrast between the target lesions and the surrounding anatomic landmarks $[22,41]$.

To overcome the limitations of EM tracking-based fusion imaging, respiratory gating or motion correction by the dynamic reference position sensor placed on the patient would be helpful in minimizing the registration error due to the movement of the patient or of the devices [3]. In addition, we hope that real-time non-rigid registration that can provide localized stretching and deformation of the fused CT or MR images [42] and thus, expected to compensate for liver deformation occurring on real-time US, will be available in the near future.

\section{Summary}

Fusion imaging is a technique that fuses the working data set and the reference data sets. With the fused reference data sets, fusion imaging is useful for localizing inconspicuous focal hepatic lesions on B-mode US. It can facilitate accurate interventional procedures such as RFA and biopsy even for challenging target lesions.

ORCID: Min Woo Lee: http://orcid.org/0000-0001-9048-9011

\section{Conflict of Interest}

No potential conflict of interest relevant to this article was reported.

\section{Supplementary Material}

Video clip 1. Fusion imaging of real-time ultrasonography (US) and magnetic resonance (MR) images. After applying fusion imaging, real-time US and fused computed tomography (CT) or MR images are displayed simultaneously. Therefore, while we are performing real-time US, the fused CT or MR image shows the same plane and moves synchronously (http://dx.doi.org/10.14366/usg.14021.v001).

Video clip 2. Fusion with 3-dimensional (3D) ultrasonography (US)-guided radiofrequency ablation. Due to the subphrenic location of the tumor, artificial ascites was introduced into the abdominal cavity. After the insertion of three electrodes into the tumor, 3D US was obtained by scanning the liver in a sweeping manner with the patients in a breath-holding state. The 3D US volume data was fused with real-time $2 \mathrm{D} U \mathrm{~S}$, and the two image sets were displayed simultaneously on a split-screen display. In the video clip obtained during ablation, it can be seen that the ablative margin seems to be sufficient (http://dx.doi.org/10.14366/usg.14021.v002).

Video clip 3. Contrast-enhanced ultrasonography (CEUS) combined with fusion imaging for radiofrequency ablation. On CEUS, arterial enhancement is noted at a different site, the slightly upper side of the low echoic lesion, which was considered to be the index tumor on fusion imaging before contrast injection. Therefore, mistargeting was avoided owing to CEUS (http://dx.doi.org/10.14366/usg.14021. v003).

\section{References}

1. Rhim H, Lee MH, Kim YS, Choi D, Lee WJ, Lim HK. Planning sonography to assess the feasibility of percutaneous radiofrequency ablation of hepatocellular carcinomas. AJR Am J Roentgenol 2008; 190:1324-1330.

2. Lencioni $R$, Cioni $D$, Crocetti L, Franchini $C$, Pina $C D$, Lera J, et al. Early-stage hepatocellular carcinoma in patients with cirrhosis: long-term results of percutaneous image-guided radiofrequency ablation. Radiology 2005;234:961-967.

3. Maybody M, Stevenson C, Solomon SB. Overview of navigation systems in image-guided interventions. Tech Vasc Interv Radiol 2013;16:136-143.

4. Lee MW, Lim HK, Kim YJ, Choi D, Kim YS, Lee WJ, et al. Percutaneous sonographically guided radio frequency ablation of hepatocellular carcinoma: causes of mistargeting and factors affecting the feasibility of a second ablation session. J Ultrasound Med 2011;30: 607-615.

5. Lee MW, Rhim H, Cha DI, Kim YJ, Lim HK. Planning US for percutaneous radiofrequency ablation of small hepatocellular carcinomas (1-3 cm): value of fusion imaging with conventional US and CT/MR images. J Vasc Interv Radiol 2013;24:958-965.

6. Park HJ, Lee MW, Lee MH, Hwang J, Kang TW, Lim S, et al. Fusion imaging-guided percutaneous biopsy of focal hepatic lesions with poor conspicuity on conventional sonography. J Ultrasound Med 2013;32:1557-1564.

7. Ewertsen C, Saftoiu A, Gruionu LG, Karstrup S, Nielsen MB. Realtime image fusion involving diagnostic ultrasound. AJR Am J Roentgenol 2013;200:W249-W255.

8. Abi-Jaoudeh N, Kruecker J, Kadoury S, Kobeiter H, Venkatesan $A M$, Levy $E$, et al. Multimodality image fusion-guided procedures: technique, accuracy, and applications. Cardiovasc Intervent Radiol 2012;35:986-998.

9. Song KD, Lee MW, Rhim H, Cha DI, Chong Y, Lim HK. Fusion imaging-guided radiofrequency ablation for hepatocellular carcinomas not visible on conventional ultrasound. AJR Am J Roentgenol 2013;201:1141-1147. 
10. Wein W, Brunke S, Khamene A, Callstrom MR, Navab N. Automatic CT-ultrasound registration for diagnostic imaging and imageguided intervention. Med Image Anal 2008;12:577-585.

11. Nam WH, Kang DG, Lee $D$, Lee JY, Ra JB. Automatic registration between 3D intra-operative ultrasound and pre-operative CT images of the liver based on robust edge matching. Phys Med Biol 2012;57:69-91.

12. Clevert DA, Paprottka PM, Helck A, Reiser M, Trumm CG. Image fusion in the management of thermal tumor ablation of the liver. Clin Hemorheol Microcirc 2012;52:205-216.

13. Crocetti $L$, Lencioni $R$, Debeni $S$, See TC, Pina CD, Bartolozzi C. Targeting liver lesions for radiofrequency ablation: an experimental feasibility study using a CT-US fusion imaging system. Invest Radiol 2008;43:33-39.

14. Lee JY, Choi BI, Chung YE, Kim MW, Kim SH, Han JK. Clinical value of CT/MR-US fusion imaging for radiofrequency ablation of hepatic nodules. Eur J Radiol 2012;81:2281-2289.

15. Min JH, Lee MW, Rhim H, Cha DI, Lim S, Choi SY, et al. Local tumour progression after loco-regional therapy of hepatocellular carcinomas: value of fusion imaging-guided radiofrequency ablation. Clin Radiol 2014;69:286-293.

16. Makino Y, Imai Y, Ohama H, Igura T, Kogita S, Sawai Y, et al. Ultrasonography fusion imaging system increases the chance of radiofrequency ablation for hepatocellular carcinoma with poor conspicuity on conventional ultrasonography. Oncology 2013;84 Suppl 1:44-50.

17. Kim YJ, Lee MW, Park HS. Small hepatocellular carcinomas: ultrasonography guided percutaneous radiofrequency ablation. Abdom Imaging 2013;38:98-111.

18. Minami Y, Chung H, Kudo M, Kitai S, Takahashi S, Inoue T, et al. Radiofrequency ablation of hepatocellular carcinoma: value of virtual CT sonography with magnetic navigation. AJR Am J Roentgenol 2008;190:W335-W341.

19. Kitada T, Murakami T, Kuzushita N, Minamitani K, Nakajo K, Osuga $K$, et al. Effectiveness of real-time virtual sonography-guided radiofrequency ablation treatment for patients with hepatocellular carcinomas. Hepatol Res 2008;38:565-571.

20. Minami Y, Kudo M, Chung H, Inoue T, Takahashi S, Hatanaka K, et al. Percutaneous radiofrequency ablation of sonographically unidentifiable liver tumors: feasibility and usefulness of a novel guiding technique with an integrated system of computed tomography and sonographic images. Oncology 2007;72 Suppl 1:111-116.

21. Hirooka M, luchi $H$, Kumagi $T$, Shigematsu $S$, Hiraoka $A$, Uehara $T$, et al. Virtual sonographic radiofrequency ablation of hepatocellular carcinoma visualized on CT but not on conventional sonography. AJR Am J Roentgenol 2006;186:S255-S260.

22. Lee MW, Rhim H, Cha DI, Kim YJ, Choi D, Kim YS, et al. Percutaneous radiofrequency ablation of hepatocellular carcinoma: fusion imaging guidance for management of lesions with poor conspicuity at conventional sonography. AJR Am J Roentgenol 2012;198:1438-1444.

23. Venkatesan AM, Kadoury S, Abi-Jaoudeh N, Levy EB, MaassMoreno R, Krucker J, et al. Real-time FDG PET guidance during biopsies and radiofrequency ablation using multimodality fusion with electromagnetic navigation. Radiology 2011;260:848-856

24. Krucker J, Xu S, Venkatesan A, Locklin JK, Amalou H, Glossop N, et al. Clinical utility of real-time fusion guidance for biopsy and ablation. J Vasc Interv Radiol 2011;22:515-524.

25. Lee MW, Kim YJ, Park HS, Yu NC, Jung SI, Ko SY, et al. Targeted sonography for small hepatocellular carcinoma discovered by $\mathrm{CT}$ or MRI: factors affecting sonographic detection. AJR Am J Roentgenol 2010;194:W396-W400.

26. Woo S, Lee JM, Yoon JH, Joo I, Kim SH, Lee JY, et al. Smalland medium-sized hepatocellular carcinomas: monopolar radiofrequency ablation with a multiple-electrode switching systemmid-term results. Radiology 2013;268:589-600.

27. Masuzaki R, Shiina S, Tateishi R, Yoshida H, Goto E, Sugioka Y, et al. Utility of contrast-enhanced ultrasonography with Sonazoid in radiofrequency ablation for hepatocellular carcinoma. J Gastroenterol Hepatol 2011;26:759-764.

28. Kudo M, Hatanaka K, Maekawa K. Newly developed novel ultrasound technique, defect reperfusion ultrasound imaging, using sonazoid in the management of hepatocellular carcinoma. Oncology 2010;78 Suppl 1:40-45.

29. Numata K, Fukuda H, Morimoto M, Kondo M, Nozaki A, Oshima $T$, et al. Use of fusion imaging combining contrast-enhanced ultrasonography with a perflubutane-based contrast agent and contrast-enhanced computed tomography for the evaluation of percutaneous radiofrequency ablation of hypervascular hepatocellular carcinoma. Eur J Radiol 2012;81:2746-2753.

30. Maruyama H, Takahashi M, Ishibashi H, Okugawa H, Okabe S, Yoshikawa $M$, et al. Ultrasound-guided treatments under low acoustic power contrast harmonic imaging for hepatocellular carcinomas undetected by B-mode ultrasonography. Liver Int 2009;29:708-714.

31. Minami Y, Kudo M. Review of dynamic contrast-enhanced ultrasound guidance in ablation therapy for hepatocellular carcinoma. World J Gastroenterol 2011;17:4952-4959.

32. Kudo M, Hatanaka K, Maekawa K. Defect reperfusion imaging, a newly developed novel technology using Sonazoid in the treatment of hepatocellular carcinoma. J Med Ultrasound 2008;16:169-176.

33. Min JH, Lim HK, Lim S, Kang TW, Song KD, Choi SY, et al. Radiofrequency ablation of very-early-stage hepatocellular carcinoma inconspicuous on fusion imaging with B-mode US: value of fusion imaging with contrast-enhanced US. Clin Mol Hepatol 2014;20:61-70.

34. Hakime A, Deschamps F, De Carvalho EG, Barah A, Auperin A, De 
Baere T. Electromagnetic-tracked biopsy under ultrasound guidance: preliminary results. Cardiovasc Intervent Radiol 2012;35:898-905.

35. Tomonari A, Tsuji K, Yamazaki H, Aoki H, Kang JH, Kodama Y, et al. Feasibility of the virtual needle tracking system for percutaneous radiofrequency ablation of hepatocellular carcinoma. Hepatol Res 2013;43:1352-1355.

36. Hakime A, Barah A, Deschamps F, Farouil G, Joskin J, Tselikas L, et al. Prospective comparison of freehand and electromagnetic needle tracking for US-guided percutaneous liver biopsy. J Vasc Interv Radiol 2013;24:1682-1689.

37. Ewertsen C, Nielsen KR, Nielsen MB. Freehand biopsy guided by electromagnetic needle tracking: a phantom study. Ultraschall Med 2011;32:614-618.

38. Hakime A, Deschamps F, De Carvalho EG, Teriitehau C, Auperin A, De Baere T. Clinical evaluation of spatial accuracy of a fusion imaging technique combining previously acquired computed tomography and real-time ultrasound for imaging of liver metastases. Cardiovasc Intervent Radiol 2011;34:338-344.

39. Lim S, Lee MW, Rhim H, Cha DI, Kang TW, Min JH, et al. Mistargeting after fusion imaging-guided percutaneous radiofrequency ablation of hepatocellular carcinomas. J Vasc Interv Radiol 2014;25:307314.

40. Ward TJ, Goldman RE, Weintraub JL. Electromagnetic navigation with multimodality image fusion for image-guided percutaneous interventions. Tech Vasc Interv Radiol 2013;16:177-181.

41. Kunishi Y, Numata K, Morimoto M, Okada M, Kaneko T, Maeda $S$, et al. Efficacy of fusion imaging combining sonography and hepatobiliary phase MRI with Gd-EOB-DTPA to detect small hepatocellular carcinoma. AJR Am J Roentgenol 2012;198:106114.

42. Lee $D$, Nam WH, Lee JY, Ra JB. Non-rigid registration between 3D ultrasound and CT images of the liver based on intensity and gradient information. Phys Med Biol 2011;56:117-137. 